\title{
Density-wave phases of dipolar fermions in a bilayer
}

\author{
F. M. Marchetti ${ }^{1}$ and M. M. Parish ${ }^{2}$ \\ ${ }^{1}$ Departamento de Física Teórica de la Materia Condensada, Universidad Autónoma de Madrid, Madrid 28049, Spain \\ ${ }^{2}$ London Centre for Nanotechnology, Gordon Street, London WC1H OAH, United Kingdom
}

(Received 20 July 2012; published 10 January 2013)

\begin{abstract}
We investigate the phase diagram of dipolar fermions with aligned dipole moments in a two-dimensional (2D) bilayer. Using a version of the Singwi-Tosi-Land-Sjölander scheme recently adapted to dipolar fermions in a single layer [M. M. Parish and F. M. Marchetti, Phys. Rev. Lett. 108, 145304 (2012)], we determine the density-wave instabilities of the bilayer system within linear response theory. We find that the bilayer geometry can stabilize the collapse of the 2D dipolar Fermi gas with intralayer attraction to form a new density wave phase that has an orientation perpendicular to the density wave expected for strong intralayer repulsion. We thus obtain a quantum phase transition between stripe phases that is driven by the interplay between strong correlations and the architecture of the low-dimensional system.
\end{abstract}

DOI: 10.1103/PhysRevB.87.045110

PACS number(s): 67.85.Lm, 71.45.Gm, 71.45.Lr

Density-wave phases such as stripes are apparently ubiquitous in nature. They are typically found in quasi-twodimensional or layered materials, ${ }^{1-3}$ where they manifest as periodic modulations of the electron density within the two-dimensional (2D) layers. Moreover, such stripes have even been observed in high-temperature superconductors. ${ }^{4,5}$ However, despite their ubiquity and potential importance, their origins and behavior are still under debate. Indeed, a central question is whether stripes are driven by electron-electron repulsion or simply by distortions of the underlying crystal structure. ${ }^{6}$

One route to gaining insight into the problem is to study cleaner, more tunable analogs of these electron systems. Quantum degenerate Fermi gases with long-range dipolar interactions $\mathrm{s}^{7,8}$ provide just such a system in which to investigate density-wave phases. Such dipolar Fermi gases have recently been realized experimentally with both magnetic atoms $^{9}$ and polar diatomic molecules. ${ }^{10-12}$ In particular, ultracold polar molecules of ${ }^{40} \mathrm{~K}{ }^{87} \mathrm{Rb}$ have been confined to $2 \mathrm{D}$ layers using an optical lattice, ${ }^{13}$ thus paving the way for exploring long-range interactions in low-dimensional systems.

For a 2D gas of polar molecules, the dipole-dipole interactions can be controlled by aligning the dipole moments with an external electric field. For small dipole tilt angles $\theta$ with respect to the plane normal, the dipolar interactions are purely repulsive, while for $\theta \gtrsim \pi / 4$, the interactions acquire a significant attractive component such that the dipolar Fermi system is unstable towards collapse for sufficiently strong interactions. ${ }^{14-17}$ Away from collapse, in the repulsive regime, previous theoretical work has predicted the existence of a stripe phase, ${ }^{15-18}$ even for the case where the dipolar interactions are isotropic $(\theta=0)$ and the system must spontaneously break rotational symmetry. ${ }^{16}$ Here we investigate the effect of the low-dimensional architecture on density instabilities by considering dipolar fermions in a $2 \mathrm{D}$ bilayer geometry.

We determine the phase diagram of the bilayer system within linear response theory, using a version of the SingwiTosi-Land-Sjölander (STLS) scheme ${ }^{19}$ recently developed in Reference 16. Based on this analysis, we show that the bilayer geometry can actually stabilize the collapse of the 2D Fermi gas to form a new density wave (Fig. 1). However, in contrast to the stripes in the repulsive regime, this new stripe phase has density modulations along the direction of the dipole tilt (Fig. 2) and can also be well described by a simplified STLS theory that involves exchange correlations only. Our work thus reveals a new quantum phase transition between two different stripe modulations, where one phase is driven by strong repulsive correlations and the other is driven by the bilayer architecture.

In the following, we consider the bilayer geometry shown in the insets of Fig. 2. Here, the dipole moments (of strength $D$ ) are aligned by an external electric field $\mathbf{E}$ lying in the $x-z$ plane and at angle $\theta$ with respect to the $z$ direction. We parametrize the $x-y$ in-plane momentum by polar coordinates $\mathbf{q}=(q, \phi)$, with $\phi=0$ corresponding to the direction $x$ of the dipole tilt. The remaining system parameters are the bilayer distance $d$ and the Fermi wave vector $k_{F}=\sqrt{4 \pi n}$ ( $n$ is the density in each layer). For dipoles confined in a layer of width $W$, in the limit $q W \ll 1$, the effective $2 \mathrm{D}$ intralayer interaction can be written as ${ }^{20}$

$$
v_{11}(\mathbf{q})=V_{0}-2 \pi D^{2} q \xi(\theta, \phi),
$$

where $\xi(\theta, \phi)=\cos ^{2} \theta-\sin ^{2} \theta \cos ^{2} \phi$ and $V_{0}$ is the $W$ dependent short-ranged contact interaction. The confinement width $W$ provides a natural cut-off for the quasi-2D system: $\Lambda \sim 1 / W \gg k_{F}$.

Likewise, in the limit $W \ll d$, we can write the interlayer interaction as ${ }^{21}$

$$
v_{12}(\mathbf{q})=-2 \pi D^{2} q e^{-q d}[\xi(\theta, \phi)+i \sin 2 \theta \cos \phi] .
$$

Note that for $\theta \neq 0$, this interaction is complex and satisfies $v_{21}(\mathbf{q})=v_{12}^{*}(\mathbf{q})=v_{12}(-\mathbf{q})$. This arises from the fact that the interlayer interaction in real space is not invariant under the transformation $\mathbf{r} \mapsto-\mathbf{r}$.

Assuming identical layers, one can parametrize the bilayer system using only three dimensionless quantities: the tilt angle $\theta$, the bilayer distance $k_{F} d$, and the interaction strength $U=$ $m D^{2} k_{F} / \hbar^{2}$, with $m$ being the fermion mass. The cut-off $\Lambda$ and the contact interaction $V_{0}$ should not be relevant since these do not affect the low-energy behavior of dipolar fermions, and, indeed, the procedure we employ preserves this. 


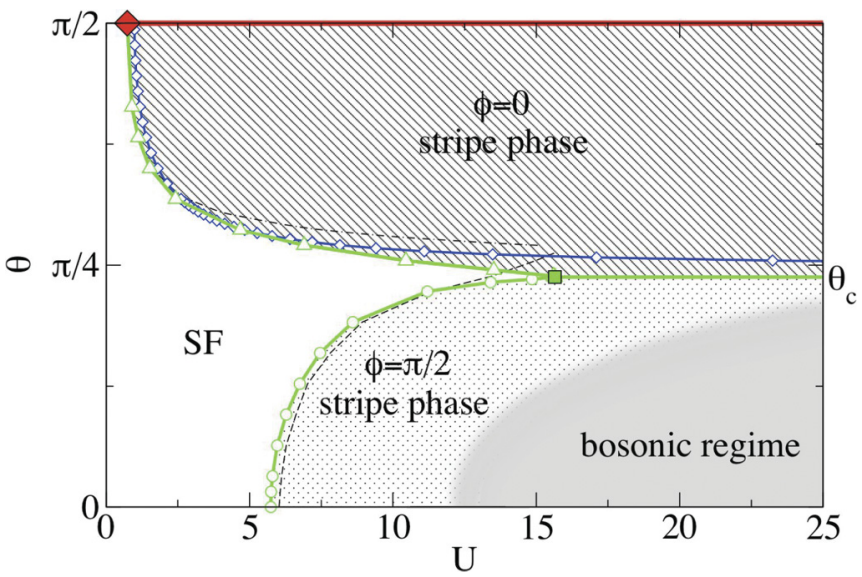

FIG. 1. (Color online) Phase diagram for a dipolar Fermi gas in a bilayer at fixed interlayer distance, $k_{F} d=2$, as a function of $\theta$ (see Fig. 2) and interaction $U=m D^{2} k_{F} / \hbar^{2}$. The liquid phase is superfluid (SF). The (green) open triangles [circles] set the boundary of the stripe phase oriented along $\phi=0[\phi=\pi / 2]$, derived from a self-consistent STLS calculation. The filled (green) square at $\theta_{c} \simeq 0.75$ and $U \simeq$ 15.65 is a quantum critical point beyond which there is a phase transition between the two stripe phases. The (blue) open diamonds for the $\phi=0$ stripe phase are instead determined including exchange correlations only (see text). These boundaries can be compared to the $\phi=\pi / 2$ stripe transition (dashed line) and the collapse instability (dashed-dotted line) for the single-layer case. ${ }^{16}$ The shaded "bosonic" region is where the system can be described in terms of interlayer bosonic dimers. The (red) filled diamond and thick (red) line at $\theta=$ $\pi / 2$ indicate collapse in the bilayer.

We now turn to the linear response theory used to analyze the inhomogeneous phases of the dipolar system. In the bilayer (and multilayers generally), the linear density response $\delta n_{i}$ to

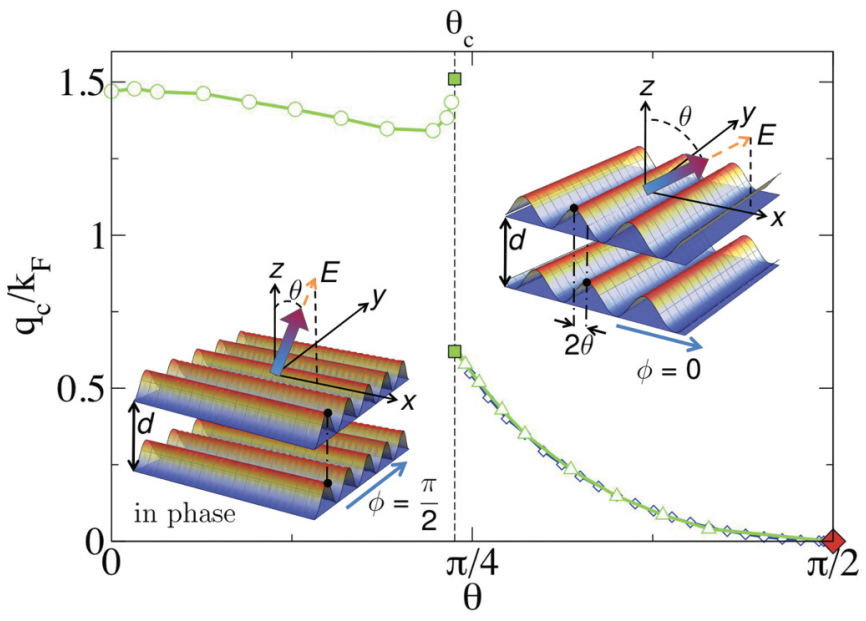

FIG. 2. (Color online) Critical wave vector $q_{c} / k_{F}$ for the $\phi=\pi / 2$ stripe phase $\left(\theta<\theta_{c}\right)$ and the $\phi=0$ one $\left(\theta>\theta_{c}\right)$; same parameters and symbol scheme as in Fig. 1. The insets depict the alignment of the dipoles with the electric field $\mathbf{E}$ and the features of the two different stripe phases. For the $\phi=0$ stripe phase, the density modulations in the two layers have a phase shift $\eta \simeq 2 \theta$, while the wave vector $q_{c}$ decreases with increasing tilt angle $\theta$ down to $q_{c}=0$ for $\theta=\pi / 2$ (filled [red] diamond), where the gas collapses. For density modulations along $\phi=\pi / 2, q_{c}$ appears to be fixed by the density. an external perturbing field $V_{i}^{\text {ext }}$ defines the density-density correlation function matrix $\chi_{i j}$,

$$
\delta n_{i}(\mathbf{q}, \omega)=\sum_{j} \chi_{i j}(\mathbf{q}, \omega) V_{j}^{\mathrm{ext}}(\mathbf{q}, \omega),
$$

where $i$ and $j$ are the layer indices. For a noninteracting gas, we clearly have $\chi_{i j}=\delta_{i j} \Pi$, where the noninteracting intralayer response function $\Pi(q, \omega)$ can be evaluated analytically. ${ }^{22}$ Typically, one includes interactions via the random phase approximation (RPA), where one uses a perturbing field that contains an effective potential due to the perturbed density: $V_{j}^{\text {ext }} \mapsto V_{j}^{\text {ext }}+\sum_{j} v_{i j} \delta n_{j}$, with intralayer potential $v_{22}(\mathbf{q})=$ $v_{11}(\mathbf{q})$. However, as has been argued recently for the singlelayer case, RPA is never accurate for dipolar interactions, since it neglects exchange correlations ${ }^{17,18}$ which are important even in the long-wavelength limit. ${ }^{16}$

A straightforward and physically motivated way of incorporating correlations beyond RPA is by means of local field factors $G_{i j}(\mathbf{q})$ (see also Ref. 23). Here, the (inverse) response function now reads

$$
\chi^{-1}{ }_{i j}(\mathbf{q}, \omega)=\frac{\delta_{i j}}{\Pi(q, \omega)}-v_{i j}(\mathbf{q})\left[1-G_{i j}(\mathbf{q})\right] .
$$

Note that we clearly recover both RPA and the noninteracting case if we take, respectively, $G_{i j}=0$ or $G_{i j}=1$. This response function can be related to the "layer-resolved" static structure factor $S_{i j}(\mathbf{q})$ by the fluctuation-dissipation theorem,

$$
S_{i j}(\mathbf{q})=-\frac{\hbar}{\pi n} \int_{0}^{\infty} d \omega \chi_{i j}(\mathbf{q}, i \omega) .
$$

In turn, we can approximate the local field factors using the STLS scheme, ${ }^{19}$

$$
G_{i j}(\mathbf{q})=\frac{1}{n} \int \frac{d \mathbf{k}}{(2 \pi)^{2}} \frac{\mathbf{q} \cdot \mathbf{k}}{q^{2}} \frac{v_{i j}(\mathbf{k})}{v_{i j}(\mathbf{q})}\left[\delta_{i j}-S_{i j}(\mathbf{q}-\mathbf{k})\right] .
$$

The response function $\chi_{i j}$ (and associated structure factor $S_{i j}$ ) can now be determined by solving Eqs. (4)-(6) selfconsistently. The STLS scheme has been heavily utilized for Coulomb interactions and it has proven to be very successful for describing the dielectric function of several strongly correlated electron systems (see Ref. 24 and references therein). Following Ref. 16, we consider an improved version of the STLS scheme that has been adapted to the dipolar system. In essence, it ensures that our results are insensitive to $\Lambda$ and $V_{0}$ by requiring that the intralayer correlations be dominated by Pauli exclusion at large wavelengths $q \gg 2 k_{F}$.

For identical layers, we can assume that $S_{22}=S_{11}$, $S_{21}=S_{12}^{*}$ (and similarly for the local field factors $G_{i j}$ ). Note that the complex form of the interlayer potential (2) means that the interlayer factors $S_{12}(\mathbf{q})$ and $G_{12}(\mathbf{q})$ are also complex. However, the symmetry $v_{12}(-\mathbf{q})=v_{12}^{*}(\mathbf{q})$ is also preserved for both factors at each iteration step of our self-consistent scheme. This guarantees that physical quantities such as the "layer-resolved" pair correlation functions, $g_{i j}(\mathbf{r})=\frac{1}{n^{2}}\left\langle\psi_{i}^{\dagger}(\mathbf{r}) \psi_{j}^{\dagger}(0) \psi_{j}(0) \psi_{i}(\mathbf{r})\right\rangle$, where

$$
g_{i j}(\mathbf{r})=1+\frac{1}{n} \int \frac{d \mathbf{q}}{(2 \pi)^{2}} e^{i \mathbf{q} \cdot \mathbf{r}}\left[S_{i j}(\mathbf{q})-\delta_{i j}\right],
$$

are always real, even when $i \neq j$. 
We determine the density instabilities of the bilayer system by analyzing the divergences of the static response function matrix $\chi_{i j}(\mathbf{q}, 0)$. Specifically, we search for zeros of the largest inverse eigenvalue,

$$
\chi_{+}^{-1}=\frac{1}{\Pi}-v_{11}\left[1-G_{11}\right]+\left|v_{12}\left[1-G_{12}\right]\right| .
$$

A zero of $\chi_{+}^{-1}(\mathbf{q}, 0)$ at a critical wave vector $\mathbf{q}_{c}$ signals an instability towards the formation of a density wave with period set by $\mathbf{q}_{c}$. If the instability occurs for a specific direction $\phi$, then the density-wave phase corresponds to a one-dimensional modulation (or stripe phase) of period $2 \pi / q_{c}$ oriented along $\phi$. In this way, we obtain the phase diagram plotted in Fig. 1 for $k_{F} d=2$.

For tilt angles $\theta<\theta_{c} \simeq 0.75$, we find a stripe phase along $\phi=\pi / 2$ that is of a similar nature to the one found in a single layer (dashed line of Fig. 1). In particular, it is driven by strong intralayer correlations induced by the repulsive part of $v_{11}$, as evidenced by the relative insensitivity of $q_{c}$ to the bilayer geometry and to $\theta$ (see Fig. 2). However, the presence of the second layer can decrease the value of the critical interaction strength $U_{c}$ for stripe formation, as one might expect from the form of Eq. (8). The attractive part of $v_{12}(\mathbf{q})$ also ensures that the density waves along $\phi=\pi / 2$ in each layer are in phase. Similar results were found using the conserving Hartree-Fock (HF) approximation ${ }^{18,25}$ but for much smaller values of $U_{c}$, like in the single-layer case. The shift of $U_{c}$ due to the other layer is relatively small for distance $k_{F} d=2$ (see Fig. 1 at small values of $\theta$ ), but it can become substantial for smaller $k_{F} d$ since Eq. (8) depends exponentially on the bilayer distance. However, for smaller distances, we then encounter phases involving strong interlayer pairing ${ }^{26-28}$ and the system would instead be better described in terms of interlayer bosonic dimers, as we discuss later.

In the isotropic case $(\theta=0)$, we find that the system spontaneously breaks rotational symmetry to form a stripe phase at $U \simeq 5.74$, similarly to the single-layer case. ${ }^{16}$ One can only observe this symmetry breaking at $\theta=0$ by starting the STLS iteration with a solution for small but finite $\theta$. This effectively corresponds to taking the limit $\theta \rightarrow 0$, which is somewhat akin to classical ferromagnetism, where one must consider the limit where magnetic field goes to zero. This stripe phase precedes Wigner crystallization which, according to quantum Monte Carlo (QMC) calculations, occurs at $U \simeq 25$ for perpendicular fermionic dipoles in a single layer. ${ }^{29}$

For $\theta>\arcsin (1 / \sqrt{3})$, the intralayer interaction develops an attractive sliver in the plane that can eventually lead to collapse in the single layer. ${ }^{14-17}$ Here, for large-enough $U$ and $\theta$, the attraction overcomes Pauli exclusion and the compressibility of the gas goes to zero $\left[\chi_{+}^{-1}(\mathbf{q} \rightarrow 0,0)=0\right]$. However, we find that the bilayer geometry can actually stabilize the collapse to form a new density-wave phase that is oriented along the $\phi=0$ direction (Fig. 1). Referring to Fig. 2, we see that this stripe phase has a longer wavelength than the $\phi=\pi / 2$ one and is dependent on the system geometry. Indeed, we find that $q_{c}$ smoothly decreases with increasing $\theta$, reaching $q_{c}=0$ at $\theta=\pi / 2$, where the intralayer attraction always appears to cause collapse at a fixed $U_{c}$. Away from $\theta=\pi / 2$, we find that the $\phi=0$ stripe phase has $q_{c} \sim 1 / d$ in the limit $d \rightarrow \infty$, which is reminiscent of the behavior of charge density waves in electron-hole bilayers.

The $\phi=0$ stripe also features a nontrivial phase shift $\eta$ between the density waves in each layer. At the stripe transition, it can be shown that

$$
e^{i \eta}=-\frac{v_{12}(\mathbf{q})\left[1-G_{12}(\mathbf{q})\right]}{\left|v_{12}(\mathbf{q})\left[1-G_{12}(\mathbf{q})\right]\right|} .
$$

When $v_{12}$ and $G_{12}$ are real, like for the $\phi=\pi / 2$ stripe phase, then $e^{i \eta}=1$ and the density waves in each layer are in phase, as mentioned previously. However, $v_{12}$ is complex for the $\phi=0$ stripe phase and, thus, the density waves are generally shifted with respect to one another. Indeed, as shown below, the interlayer correlations are small in this phase, i.e., $\left|G_{12}\right| \ll 1$; therefore, the phase shift corresponds to $\eta \simeq 2 \theta$ (see insets of Fig. 2) and is essentially independent of $k_{F} d$.

The existence of two stripe phases leads to a new quantum phase transition where the stripes change their orientation. In Fig. 1, this occurs beyond the critical point $\theta_{c} \simeq 0.75$ and $U_{c} \simeq 15.65$ where the two stripe phase boundaries meet. Here, when $k_{F} d$ is fixed, the transition can be accessed by changing the tilt angle $\theta$. Alternatively, one can fix $\theta \lesssim \pi / 4$, which is below the onset of collapse in the single layer, and vary $k_{F} d$, since we expect the critical angle $\theta_{c}$ to decrease with decreasing $k_{F} d$. Eventually, at $k_{F} d \simeq 1$, one enters the regime where the physics of bosonic interlayer dimers dominates.

Further insight into the stripe phases can be gained by examining the intra- and interlayer pair correlation functions $g_{i j}(\mathbf{r})$ on the liquid (superfluid) side of the transition. For the $\phi=0$ stripe phase (bottom panel of Fig. 3), we find that neither pair correlation function changes significantly as we approach the transition. In particular, $g_{11}(\mathbf{r})$ only deviates slightly from the noninteracting case $(U=0)$, while $g_{12}(\mathbf{r})$ slowly oscillates close to 1 , indicating that interlayer correlations are small, i.e., $\left|G_{12}\right| \ll 1$. This suggests that we can accurately model the

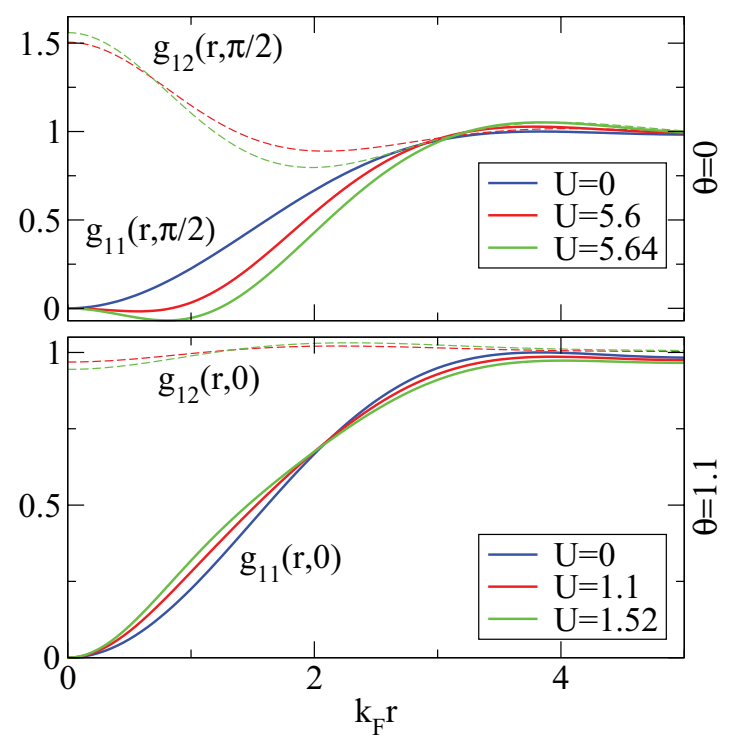

FIG. 3. (Color online) Intra- and interlayer pair correlation functions $g_{i j}(\mathbf{r})$ for increasing values of the interaction strength $U$ towards the $\phi=\pi / 2$ stripe phase ( $\theta=0$ top panel) and the $\phi=0$ phase $(\theta=1.1 \simeq 0.35 \pi$ bottom panel $)$. 
$\phi=0$ stripe phase using exchange correlations only. To this end, we construct a simplified STLS theory where we take $G_{12}(\mathbf{q})=0$ and then determine the intralayer local field factor $G_{11}(\mathbf{q})$ by feeding the noninteracting intralayer structure factor $S_{0}(q)=-\frac{\hbar}{\pi n} \int_{0}^{\infty} d \omega \Pi(q, \omega)$ into Eq. (6). We then evaluate the phase boundary for the $\phi=0$ stripe within this simplified HF theory. Referring to Figs. 1 and 2, we see that we obtain very good agreement with the full STLS calculation, particularly when $U$ and $\theta$ are not too large so the intralayer $p$-wave pairing correlations are expected to be weakest. ${ }^{14,17}$ In addition, the collapse instability at $\theta=\pi / 2$ is unaffected by the other layer since the interlayer Hartree term is zero for $\mathbf{q}=0$. We expect one can obtain quantitatively similar results for the $\phi=0$ stripe phase using the conserving HF approximation. ${ }^{30}$

By contrast, for the $\phi=\pi / 2$ stripe phase (top panel of Fig. 3), we see that correlations beyond exchange become substantial, resulting in a pronounced "correlation hole" for $g_{11}(\mathbf{r})$ with increasing interaction strength, like in the singlelayer case, ${ }^{16}$ note that the STLS procedure does not guarantee that $g_{11}$ is always positive, ${ }^{24}$ and, thus, we sometimes obtain unphysical negative values. The intralayer correlations also develop a substantial $\phi$ anisotropy as we near the stripe transition. At the same time, the interlayer pair correlation function $g_{12}(\mathbf{r})$ increases at $\mathbf{r}=0$, a feature that has been ascribed to an imminent bound-state instability. ${ }^{31}$

Indeed, the attractive part of $v_{12}(\mathbf{q})$ always yields a two-body bound state composed of one fermion from each layer. $^{32,33}$ Hence, any liquid phase in the phase diagram contains pairing correlations and must therefore be superfluid (Fig. 1). When the size of these interlayer dimers $l_{B}$ is smaller than the interparticle spacing, i.e., $l_{B} \ll 1 / k_{F}$, then the system is better described in terms of bosonic dimers and our approach of analyzing density instabilities of the Fermi liquid phase is unlikely to be accurate. To estimate this region of phase space where bosonic behavior dominates, we solve the two-body problem, $E \psi_{\mathbf{k}}=\frac{\hbar^{2} \mathbf{k}^{2}}{m} \psi_{\mathbf{k}}+\int \frac{d \mathbf{k}}{(2 \pi)^{2}} v_{12}\left(\mathbf{k}-\mathbf{k}^{\prime}\right) \psi_{\mathbf{k}^{\prime}}$, where $\psi_{\mathbf{k}}$ is the two-body wave function in terms of relative coordinates and $E$ is the dimer binding energy. We estimate the dimer size as $l_{B} \sim \hbar / \sqrt{m|E|}$ and then determine the "critical" line $k_{F} l_{B}=1$ for the bosonic regime, as plotted in Fig. 1 (shaded region). We see that this region is well separated from the stripe phase boundaries. Thus, we expect pairing correlations to be small at the stripe transition and, therefore, our results to be reasonable for $k_{F} d=2$.

Note that the presence of bosonic dimers hastens the onset Wigner crystallization: QMC calculations ${ }^{34,35}$ predict that perpendicularly aligned bosons will crystallize at $U \simeq 8$. For increasing $\theta$, the interlayer dimer becomes more weakly bound until eventually the fermions preferentially form pairs within the same layer instead. With decreasing $k_{F} d$, however, the regime of interlayer bosons expands so it encroaches on our predicted stripe transitions for $k_{F} d \simeq 1$ and takes us beyond the scope of this article.

Our predicted stripe phases should be accessible experimentally with cold dipolar gases. In particular, the bilayer distance $k_{F} d=2$ can be achieved for a typical 2D density $n \sim 1.3 \times 10^{8} \mathrm{~cm}^{-2}$ and layer spacing $d=500 \mathrm{~nm}$. Polar molecules such as $\mathrm{LiCs}^{8}$ have dipolar moments $D \sim 0.35-$ $1.3 \mathrm{D}$ (corresponding to $U \sim 1-14$ ), which allow one to explore both $\phi=0$ and $\phi=\pi / 2$ stripe phases. Furthermore, the newly explored $\mathrm{NaK}$ molecules ${ }^{12}$ allow one to reach even larger values of the interaction strength $(D \sim 2.7 \mathrm{D}$ and $U \sim 28)$.

We are grateful to G. Bruun, J. Levinsen, P. Littlewood, and N. Zinner for useful discussions. F.M.M. acknowledges financial support from the Ramón y Cajal and Intelbiomat (ESF) programs, and M.M.P. acknowledges support from the EPSRC under Grant No. EP/H00369X/2. We also acknowledge the TCM group in Cambridge for hospitality.
${ }^{1}$ G. Grüner, Rev. Mod. Phys. 60, 1129 (1988).

${ }^{2}$ C. Howald, H. Eisaki, N. Kaneko, and A. Kapitulnik, Proc. Natl Acad. Sci. USA 100, 9705 (2003).

${ }^{3}$ K. C. Rahnejat, C. A. Howard, N. E. Shuttleworth, K. I. S. R. Schofield, C. F. Hirjibehedin, C. Renner, G. Aeppli, and M. Ellerby, Nat. Commun. 2, 558 (2011).

${ }^{4}$ S. A. Kivelson, E. Fradkin, and V. J. Emery, Nature 393, 550 (1998). ${ }^{5}$ S. A. Kivelson, I. P. Bindloss, E. Fradkin, V. Oganesyan, J. M. Tranquada, A. Kapitulnik, and C. Howald, Rev. Mod. Phys. 75, 1201 (2003).

${ }^{6}$ M. D. Johannes and I. I. Mazin, Phys. Rev. B 77, 165135 (2008).

${ }^{7}$ M. A. Baranov, Phys. Rep. 464, 71 (2008).

${ }^{8}$ L. D. Carr, D. DeMille, R. V. Krems, and J. Ye, New J. Phys. 11, 055049 (2009).

${ }^{9}$ M. Lu, N. Q. Burdick, and B. L. Lev, Phys. Rev. Lett. 108, 215301 (2012)

${ }^{10}$ K.-K. Ni, S. Ospelkaus, M. H. G. de Miranda, A. Pe'er, B. Neyenhuis, J. J. Zirbel, S. Kotochigova, P. S. Julienne, D. S. Jin, and J. Ye, Science 322, 231 (2008).

${ }^{11}$ M.-S. Heo, T. T. Wang, C. A. Christensen, T. M. Rvachov, D. A. Cotta, J.-H. Choi, Y.-R. Lee, and W. Ketterle, Phys. Rev. A 86, 021602(R) (2012).
${ }^{12}$ C.-H. Wu, J. W. Park, P. Ahmadi, S. Will, and M. W. Zwierlein, Phys. Rev. Lett. 109, 085301 (2012).

${ }^{13}$ M. H. G. de Miranda, A. Chotia, B. Neyenhuis, D. Wang, G. Quéméner, S. Ospelkaus, J. L. Bohn, J. Ye, and D. S. Jin, Nat. Phys. 7, 502 (2011).

${ }^{14}$ G. M. Bruun and E. Taylor, Phys. Rev. Lett. 101, 245301 (2008).

${ }^{15}$ Y. Yamaguchi, T. Sogo, T. Ito, and T. Miyakawa, Phys. Rev. A 82, 013643 (2010)

${ }^{16}$ M. M. Parish and F. M. Marchetti, Phys. Rev. Lett. 108, 145304 (2012).

${ }^{17}$ L. M. Sieberer and M. A. Baranov, Phys. Rev. A 84, 063633 (2011).

${ }^{18}$ M. Babadi and E. Demler, Phys. Rev. B 84, 235124 (2011).

${ }^{19}$ K. S. Singwi, M. P. Tosi, R. H. Land, and A. Sjölander, Phys. Rev. 176, 589 (1968).

${ }^{20}$ U. R. Fischer, Phys. Rev. A 73, 031602 (2006).

${ }^{21}$ Q. Li, E. H. Hwang, and S. Das Sarma, Phys. Rev. B 82, 235126 (2010).

${ }^{22}$ F. Stern, Phys. Rev. Lett. 18, 546 (1967).

${ }^{23}$ N. Zinner and G. Bruun, Eur. Phys. J. D 65, 133 (2011). 
${ }^{24}$ G. F. Giuliani and G. Vignale, Quantum Theory of the Electron Liquid (Cambridge University Press, Cambridge, UK, 2005).

${ }^{25}$ J. K. Block, N. T. Zinner, and G. M. Bruun, New J. Phys. 14, 105006 (2012).

${ }^{26}$ A. Pikovski, M. Klawunn, G. V. Shlyapnikov, and L. Santos, Phys. Rev. Lett. 105, 215302 (2010).

${ }^{27}$ N. T. Zinner, B. Wunsch, D. Pekker, and D.-W. Wang, Phys. Rev. A 85, 013603 (2012).

${ }^{28}$ M. A. Baranov, A. Micheli, S. Ronen, and P. Zoller, Phys. Rev. A 83, 043602 (2011).

${ }^{29}$ N. Matveeva and S. Giorgini, Phys. Rev. Lett. 109, 200401 (2012).
${ }^{30}$ Note that the $\phi=0$ stripe phase was not observed in Ref. 25 since they focused on the $\phi=\pi / 2$ instability and ignored the imaginary part of $v_{12}(\mathbf{q})$.

${ }^{31}$ L. Liu, L. Swierkowski, and D. Neilson, Physica B 249-251, 594 (1998).

${ }^{32}$ M. Klawunn, A. Pikovski, and L. Santos, Phys. Rev. A 82, 044701 (2010).

${ }^{33}$ A. G. Volosniev, N. T. Zinner, D. V. Fedorov, A. S. Jensen, and B. Wunsch, J. Phys. B 44, 125301 (2011).

${ }^{34}$ G. E. Astrakharchik, J. Boronat, I. L. Kurbakov, and Y. E. Lozovik, Phys. Rev. Lett. 98, 060405 (2007).

${ }^{35}$ H. P. Büchler, E. Demler, M. Lukin, A. Micheli, N. Prokof'ev, G. Pupillo, and P. Zoller, Phys. Rev. Lett. 98, 060404 (2007). 